TITLE:

\title{
The reduction of a quantum system of three identical particles on a plane
}

$\operatorname{AUTHOR}(\mathrm{S}):$

Iwai, T; Hirose, T

CITATION:

Iwai, T ... [et al]. The reduction of a quantum system of three identical particles on a plane. JOURNAL OF MATHEMATICAL PHYSICS 2002, 43(6): 2907-2926

ISSUE DATE:

2002-06

URL:

http://hdl.handle.net/2433/50279

\section{RIGHT:}

Copyright 2002 American Institute of Physics. This article may be downloaded for personal use only. Any other use requires prior permission of the author and the American Institute of Physics. 


\title{
The reduction of a quantum system of three identical particles on a plane
}

\author{
Toshihiro Iwai $^{\mathrm{a})}$ and Toru Hirose \\ Department of Applied Mathematics and Physics, Kyoto University, \\ Kyoto 606-8501, Japan
}

(Received 12 October 2001; accepted for publication 25 February 2002)

Quantum systems of three identical particles on a plane are analyzed from the viewpoint of symmetry. Upon reduction by rotation, such systems are described in the space of sections of a line bundle over a three-dimensional shape space whose origin represents triple collision. It is shown that if the total angular momentum is nonzero, then the wave section must vanish at the origin, while if it is zero, then the wave section can be finite at the origin. Since the particles are assumed to be identical, the quantum system admits the action of the symmetric group $S_{3}$ as well, which stands for the group of particle exchanges and is commutative with rotation. Hence the reduced system still admits the $S_{3}$ action, so that Bose and Fermi states can be discussed in the space of sections of the line bundle. A detailed analysis of a system of three free particles on a plane is presented in the latter part of the article. (C) 2002 American Institute of Physics. [DOI: 10.1063/1.1473872]

\section{INTRODUCTION}

This article deals with quantum mechanics on the center-of-mass system of three identical particles on a plane, which has manifestly two kinds of symmetries: They are rotation of all particles about the origin and particle exchanges. As is well recognized, symmetry is closely associated with the reduction of dynamical systems. One of the authors (T.I.) has already studied the reduction of quantum planar three-body systems by the use of rotational symmetry. ${ }^{1}$ As for the reduction of multiparticle systems with rotational symmetry, a point to make is the fact that the center-of-mass system is made into a principal fiber bundle with the rotation group as structure group, if the center-of-mass system is restricted to a subspace on which the rotation group acts freely. This fact was first proved by Guichardet. ${ }^{2}$ On the basis of this bundle picture, a gauge theoretical treatment becomes feasible for multiparticle systems. After Ref. 2, a number of articles $^{3-8}$ were published by T.I. for analyzing multiparticle systems in a gauge theoretical manner. The reduction method for multiparticle systems with rotational symmetry has been extended in a rather abstract way by the use of the Peter-Weyl theorem on unitary irreducible representations of compact Lie groups. ${ }^{9}$ From a physical point of view, an original article ${ }^{10}$ and a review article $^{11}$ are of great help for the gauge theoretical treatment of multiparticle systems. The gauge theoretical treatment has been given to deformable bodies as well. ${ }^{12-14}$

As is already known, ${ }^{1}$ if the triple collision of particles is excluded, the center-of-mass system for planar three bodies is diffeomorphic with $\dot{\mathrm{R}}^{4}$ and made into a principal fiber bundle $\dot{\mathrm{R}}^{4} \rightarrow \dot{\mathrm{R}}^{3}$ with structure group $\mathrm{SO}(2)$, where $\mathrm{SO}(2)$ stands for the rotational symmetry whose action is to the left, and the dot symbol indicates that the origin is removed from the space in question. Further, if all the particles are identical, it will be found that the symmetric group $S_{3}$, which stands for the symmetry of three-particle exchanges, also acts on the center-of-mass system. This action is to the right and will be shown to be expressed in terms of matrices representing the group $S_{3}$. Since the right and left actions commute, the action of $S_{3}$ will project to the factor space $\dot{\mathrm{R}}^{3} \cong \dot{\mathrm{R}}^{4} / \mathrm{SO}(2)$. In

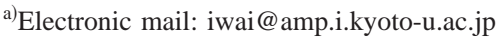


what follows, when the whole center-of-mass system is considered, the triple collision is taken into account.

On the basis of the symmetry of rotation and particle exchanges, the space of wave functions on $\mathrm{R}^{4}$ is broken up into a series of subspaces that are interpreted as eigenspaces associated with both $\mathrm{SO}(2)$ and $S_{3}$, and the time evolution of the original quantum system on $\mathrm{R}^{4}$ induces respective time evolutions in the subspaces, accordingly. The reduction to subsystems is thus accomplished. These subsystems will be identified with reduced systems to be defined on $\dot{\mathrm{R}}^{3}$ $\cong \dot{\mathrm{R}}^{4} / \mathrm{SO}(2)$ along with boundary conditions at the origin. While the theory should apply to a system containing any number of particles, and of course can do in three dimensions, too, we have chosen $n=3$ for the number of particles to present the idea in a simple form.

The organization of this paper is as follows: Section II contains a review of the center-of-mass system and of Jacobi vectors. Section III is a review of the principal bundle $\dot{R}^{4} \rightarrow \dot{R}^{3}$ with structure group $\mathrm{SO}(2)$. A connection form defined on $\dot{\mathrm{R}}^{4}$ and a metric defined on $\dot{\mathrm{R}}^{3}$ will also be reviewed, and thereby the distance function with respect to that metric on $\dot{\mathrm{R}}^{3}$ will be discussed. In Sec. IV, the action of the symmetric group $S_{3}$ on the center-of-mass system is represented explicitly in terms of matrices. Since the action of $\mathrm{SO}(2)$ and of $S_{3}$ commute, the action of $S_{3}$ on the centerof-mass system $\dot{\mathrm{R}}^{4}$ projects to $\dot{\mathrm{R}}^{3}$, which is given explicitly in Sec. V. In Sec. VI, the $L^{2}$ space of wave functions on the center-of-mass system $\mathrm{R}^{4}$ is decomposed into the sum of spaces of "equivariant" functions with respect to the $\mathrm{SO}(2)$ action on $\mathrm{R}^{4}$. If a quantum system is $\mathrm{SO}(2)$ invariant, the time evolution in the $L^{2}$ space is reduced to that in the space of equivariant functions, accordingly. In Sec. VII, interest will center on what will actually happen at the origin, the boundary of $\dot{\mathrm{R}}^{4}$, if the triple collision is taken into account. Boundary conditions for wave functions at the origin of $\mathrm{R}^{4}$ are to be considered by the use of the equivariance condition. It will be shown that according to whether the total angular momentum is nonzero or zero, the wave function vanishes at the origin or takes a finite value there. In Sec. VIII, the symmetry of particle exchanges are discussed in the space of wave functions on the center-of-mass system. The Bose and Fermi states are characterized by the respective representations of the permutation group $S_{3}$ acting on the center-of-mass system. Since the action of $\mathrm{SO}(2)$ and of $S_{3}$ commute, both the Bose and Fermi states can be constructed in the space of equivariant functions. Thus the reduction to subsystems is accomplished by the use of the symmetry of rotation and particle exchanges. Section IX deals with complex line bundles associated with the principal bundle $\dot{\mathrm{R}}^{4} \rightarrow \dot{\mathrm{R}}^{3}$. It is shown that the spaces of equivariant functions described in Sec. VI are in one-to-one correspondence with the spaces of "sections" in those complex line bundles with boundary conditions at the origin of $\mathrm{R}^{3}$. Since the action of $\mathrm{SO}(2)$ and of $S_{3}$ commute, it further turns out that the subsystems studied in Sec. VIII are indeed equivalent to quantum systems defined in the space of sections with the boundary condition at the origin along with the restriction to the Bose or Fermi state. In Sec. $\mathrm{X}$, the reduction procedure is applied to a system of free identical particles on a plane. The time evolution of the system reduces to the time evolution in the space of equivariant functions, which is expressed in the form of integral transform, and further Bose and Fermi states are formed according to the procedure developed in Sec. VIII. Section XI contains a local expression of the boundary conditions studied in Sec. VII, and also deals with a local expression of the integral transform obtained in Sec. X, to show explicitly how the reduction is accomplished indeed. Section XII contains remarks on applications to electrons on a plane.

\section{THE CONFIGURATION SPACE}

Suppose there are three particles on a plane, each with position vectors $\mathbf{x}_{j}, j=1,2,3$, and masses $m_{j}, j=1,2,3$, respectively. The set of all possible particle positions, $X$, is identified with $\mathrm{R}^{2 \times 3}$, which consists of ordered triples of position vectors $\left(\mathbf{x}_{1}, \mathbf{x}_{2}, \mathbf{x}_{3}\right)$.

Given the space $X$, one can consider two fundamental motions traced by the particles, one of which is translation and the other rotation. The space $X$ is endowed with the inner product $K: X$ $\times X \rightarrow \mathrm{R}$ which is defined by 


$$
K(x, y)=\sum_{j=1}^{3} m_{j}\left(\mathbf{x}_{j}, \mathbf{y}_{j}\right), \quad x=\left(\mathbf{x}_{1}, \mathbf{x}_{2}, \mathbf{x}_{3}\right), \quad y=\left(\mathbf{y}_{1}, \mathbf{y}_{2}, \mathbf{y}_{3}\right) \in X,
$$

where $(\mathbf{x}, \mathbf{y})$ denotes the standard inner product on $\mathrm{R}^{2}$.

Getting rid of translational degrees of freedom, we shall focus on the center-of-mass system, which is defined by

$$
X_{0}=\left\{\left(\mathbf{x}_{1}, \mathbf{x}_{2}, \mathbf{x}_{3}\right) \in X \mid \sum_{j=1}^{3} m_{j} \mathbf{x}_{j}=0\right\} .
$$

From Ref. 1, we find that $X_{0}$ has the following orthonormal basis with respect to the metric $K$ :

$$
\begin{gathered}
f_{1}=N_{1}\left(-m_{2} \mathbf{e}_{1}, m_{1} \mathbf{e}_{1}, 0\right), \\
f_{2}=N_{1}\left(-m_{2} \mathbf{e}_{2}, m_{1} \mathbf{e}_{2}, 0\right), \\
f_{3}=N_{2}\left(-m_{3} \mathbf{e}_{1},-m_{3} \mathbf{e}_{1},\left(m_{1}+m_{2}\right) \mathbf{e}_{1}\right), \\
f_{4}=N_{2}\left(-m_{3} \mathbf{e}_{2},-m_{3} \mathbf{e}_{2},\left(m_{1}+m_{2}\right) \mathbf{e}_{2}\right),
\end{gathered}
$$

where $N_{j}$ are the normalizing factors explicitly given by

$$
\begin{gathered}
N_{1}=\left(m_{1} m_{2}\left(m_{1}+m_{2}\right)\right)^{-1 / 2}, \\
N_{2}=\left(m_{3}\left(m_{1}+m_{2}\right)\left(m_{1}+m_{2}+m_{3}\right)\right)^{-1 / 2} .
\end{gathered}
$$

With respect to $f_{j}, j=1, \ldots, 4$, any $x \in X_{0}$ is represented as

$$
x=\sum_{j=1}^{4} q_{j} f_{j}, \quad q_{j}=K\left(x, f_{j}\right) .
$$

These coefficients $q_{j}$ serve as the Cartesian coordinates in $X_{0}$.

The space $X_{0}$ is isomorphic to $\mathrm{R}^{4}$ and also to $\mathrm{R}^{2} \times \mathrm{R}^{2}$, the set of pair of vectors in $\mathrm{R}^{2}$. We define the pair of two vectors as follows:

$$
\begin{gathered}
\mathbf{r}_{1}=q_{1} \mathbf{e}_{1}+q_{2} \mathbf{e}_{2}=\sqrt{\frac{m_{1} m_{2}}{m_{1}+m_{2}}}\left(\mathbf{x}_{2}-\mathbf{x}_{1}\right), \\
\mathbf{r}_{2}=q_{3} \mathbf{e}_{1}+q_{4} \mathbf{e}_{2}=\sqrt{\frac{m_{3}\left(m_{1}+m_{2}\right)}{m_{1}+m_{2}+m_{3}}}\left(\mathbf{x}_{3}-\frac{m_{1} \mathbf{x}_{1}+m_{2} \mathbf{x}_{2}}{m_{1}+m_{2}}\right) .
\end{gathered}
$$

The vectors $\mathbf{r}_{1}$ and $\mathbf{r}_{2}$ are called the Jacobi vectors, which will be effectively used in dealing with particle exchanges. Figure 1 illustrates the visual view of the Jacobi vectors, but the arrow lengths are not drawn to scale.

Both the orthonormal basis $\left\{f_{j}\right\}_{j=1, \ldots, 4}$ and the Jacobi vectors $\mathbf{r}_{1}$ and $\mathbf{r}_{2}$ are easily generalized for a planar $n$-body system, ${ }^{5}$ and for a spatial $n$-body system ${ }^{3,9}$ as well.

\section{THE INTERNAL SPACE}

Having removed the translational degrees of freedom in Sec. II, we now consider in this section the symmetry due to the rotation,

$$
x=\left(\mathbf{x}_{1}, \mathbf{x}_{2}, \mathbf{x}_{3}\right) \mapsto g x=\left(g \mathbf{x}_{1}, g \mathbf{x}_{2}, g \mathbf{x}_{3}\right), \quad g \in \mathrm{SO}(2), \quad x \in X_{0} .
$$




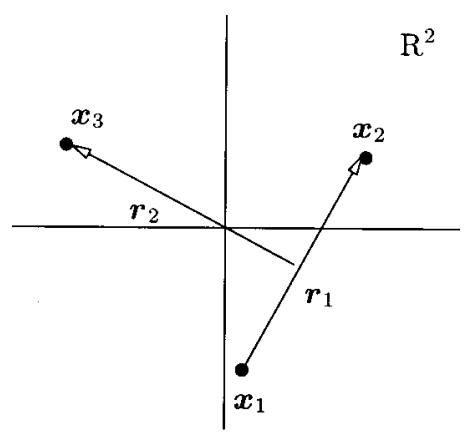

FIG. 1. Illustrating the Jacobi vectors $\mathbf{r}_{1}$ and $\mathbf{r}_{2}$ as seen in Eq. (6). $\mathbf{r}_{1}$ points along the line joining particles 1 and 2, while $\mathbf{r}_{2}$ points along the line joining particles 3 and the center-of-mass of particles 1 and 2. Note that the arrow lengths are not drawn to scale.

For a while, we forget the case where all particles collide at the origin, and consider the configuration space $\dot{X}_{0}:=X_{0}-\{0\}$. Then the $\mathrm{SO}(2)$ action becomes free. Further, the $\mathrm{SO}(2)$ action defines an equivalence relation on $\dot{X}_{0}$, and gives rise to a quotient space $\dot{X} / \mathrm{SO}(2)$. We denote by $\pi$ the natural projection from $\dot{X}_{0}$ to the quotient space,

$$
\pi: \dot{X}_{0} \rightarrow M:=\dot{X}_{0} / \mathrm{SO}(2), \quad \pi(x)=[x], \quad x \in \dot{X}_{0},
$$

where $[x]$ denotes the equivalence class of $x$. The space $M$ turns out to be a manifold which we shall call the internal or the shape space. Thus $\dot{X}_{0}$ is made into a fiber bundle with structure group $\mathrm{SO}(2){ }^{1}$

To elaborate the discussion, we give the explicit form of the projection (8). Let $q$ $=\left(q_{1}, q_{2}, q_{3}, q_{4}\right)$ denote points of space $\dot{X}_{0}$, just as was defined in (5). We notice that $X_{0} \cong \mathrm{R}^{4}$ is identified with $\mathrm{C}^{2}$ by introducing the complex variables $z_{1}, z_{2}$ through

$$
z_{1}=q_{1}+i q_{2}, \quad z_{2}=q_{3}+i q_{4}, \quad i=\sqrt{-1} .
$$

On account of $g=\left(\begin{array}{c}\cos t-\sin t \\ \sin t \cos t\end{array}\right)$, the $\mathrm{SO}(2)$ action on $\mathrm{C}^{2}$ turns out to be expressed as

$$
z=\left(z_{1}, z_{2}\right) \mapsto\left(e^{i t} z_{1}, e^{i t} z_{2}\right)=e^{i t} z .
$$

With the identification $X_{0} \cong \mathrm{C}^{2}$, the natural projection $\pi$ is realized as

$$
\pi:\left(z_{1}, z_{2}\right) \mapsto\left(\xi_{1}, \xi_{2}, \xi_{3}\right),
$$

where

$$
\xi_{1}+i \xi_{2}=2 z_{1} \bar{z}_{2}, \quad \xi_{3}=\left|z_{1}\right|^{2}-\left|z_{2}\right|^{2} .
$$

Note here that

$$
\sqrt{\sum_{k=1}^{3} \xi_{k}^{2}}=\sum_{j=1}^{4} q_{j}^{2} .
$$

It can be verified that the shape space $M$ is diffeomorphic with $\dot{\mathrm{R}}^{3}:=\mathrm{R}^{3}-\{0\},^{1}$

$$
M:=\dot{X}_{0} / \mathrm{SO}(2) \cong \dot{\mathrm{R}}^{3} .
$$

Thus the rotational degree of freedom is removed to provide the shape space $M$. 
In the remainder of this section, we make a review of the connection defined on the $\mathrm{SO}(2)$ bundle $\dot{X}_{0} \rightarrow M$ and of the metric defined on $M$. A one-form $\omega$ defined to be

$$
\omega=\frac{1}{\sum_{j=1}^{4} q_{j}^{2}}\left(-q_{2} d q_{1}+q_{1} d q_{2}-q_{4} d q_{3}+q_{3} d q_{4}\right)
$$

is called a connection form on the $\mathrm{SO}(2)$ bundle $\dot{X}_{0} \rightarrow M$. The connection form gives rise to a direct sum decomposition of the tangent space $T_{x}\left(\dot{X}_{0}\right)$ at each point $x$ of $\dot{X}_{0}$,

$$
T_{x}\left(\dot{X}_{0}\right)=V_{x} \oplus H_{x}, \quad H_{x}:=\operatorname{ker} \omega_{x}, \quad V_{x}:=T_{x}\left(\mathcal{O}_{x}\right)
$$

where $\omega_{x}$ is considered as a linear map from the tangent space $T_{x}\left(\dot{X}_{0}\right)$ to the Lie algebra $s o(2)$ $\cong \mathrm{R}$ of $\mathrm{SO}(2)$, and $T_{x}\left(\mathcal{O}_{x}\right)$ denotes the tangent space at $x$ to the $\mathrm{SO}(2)$-orbit $\mathcal{O}_{x}$ through $x \in \dot{X}_{0}$. Note here that the subspaces $H_{x}$ and $V_{x}$ are orthogonal to each other with respect to the Euclidean metric $K_{x}$ on $\dot{X}_{0}$. Since the subspace $H_{x}$ is isomorphic, as a vector space, to the tangent space $T_{\pi(x)}(M)$ to $M$ at $\pi(x)=\xi$ through the differential $\pi_{*}$ of the projection map $\pi$, and since the metric on the center-of-mass system $\dot{X}_{0}$ is invariant under the $\mathrm{SO}(2)$ action, a metric $\widetilde{K}$ on $M$ is defined through

$$
K_{x}\left(U_{1}, U_{2}\right)=\widetilde{K}_{\pi(x)}\left(\pi_{*} U_{1}, \pi_{*} U_{2}\right), \quad U_{1}, U_{2} \in H_{x} .
$$

A straightforward calculation shows that $\widetilde{K}$ is expressed as

$$
\widetilde{K}=\frac{1}{4 r} \sum_{k=1}^{3} d \xi_{k}^{2}, \quad r=\sqrt{\sum_{k=1}^{3} \xi_{k}^{2}} .
$$

By using the metric $\widetilde{K}$, we are to evaluate the distance $d_{M}\left(\xi, \xi^{\prime}\right)$ of two points $\xi$, $\xi^{\prime}$ of $M$, which will be used in Sec. XI. Since $d_{M}\left(\xi, \xi^{\prime}\right)$ is equal to the length of the geodesic joining $\xi$ to $\xi^{\prime}$, we have to find that geodesic. To this end, we first consider horizontal geodesics in $\dot{X}_{0}$ with respect to the Euclidean metric, where a curve $c(t)$ in $\dot{X}_{0}$ is, in general, called horizontal if its tangent vector $\dot{c}(t)$ is horizontal, $\dot{c}(t) \in H_{c(t)}$. We now take $z \in \pi^{-1}(\xi)$ and $w \in \pi^{-1}\left(\xi^{\prime}\right)$. The horizontal geodesic in $\dot{X}_{0}$ which projects to the geodesic in $M$ joining $\xi$ to $\xi^{\prime}$ should be a horizontal straight line joining $e^{i s} z$ to $w$ for a certain real number $s$,

$$
u(t)=w+t\left(e^{i s} z-w\right), \quad 0 \leqslant t \leqslant 1,
$$

where the parameter $s$ is to be determined so that $u(t)$ may be horizontal. By definition, the curve $u(t)$ is horizontal if and only if $\omega(\dot{u}(t))=0$. A straightforward calculation along with (15) shows that $\omega(\dot{u}(t))=0$ is equivalent to

$$
\sum_{k=1}^{2}\left(u_{k} \frac{\mathrm{d} \bar{u}_{k}}{\mathrm{~d} t}-\bar{u}_{k} \frac{\mathrm{d} u_{k}}{\mathrm{~d} t}\right)=-2 i\left|\sum_{k=1}^{2} z_{k} \bar{w}_{k}\right| \sin (s+\theta)=0,
$$

where $\theta$ is the argument of $\sum_{k=1}^{2} z_{k} \bar{w}_{k}$. Thus, $u(t)$ is horizontal if and only if $s$ is determined so as to satisfy $\sin (s+\theta)=0$ or $\cos (s+\theta)= \pm 1$. For $s$ thus determined, the horizontal straight line $u(t)$ projects to the geodesic $\pi(u(t))$ joining $\xi$ and $\xi^{\prime}$. From the definition of the metric $\widetilde{K}$, the length of $u(t), 0 \leqslant t \leqslant 1$, with respect to $K$, is equal to that of $\pi(u(t)), 0 \leqslant t \leqslant 1$, with respect to $\widetilde{K}$. The squared length of the $u(t), 0 \leqslant t \leqslant 1$ is now calculated as 


$$
\sum_{k=1}^{2}\left|e^{i s} z_{k}-w_{k}\right|^{2}=\sum_{k=1}^{2}\left|z_{k}\right|^{2}+\sum_{k=1}^{2}\left|w_{k}\right|^{2} \pm 2\left|\sum_{k=1}^{2} z_{k} \bar{w}_{k}\right|
$$

On account of the minimum property of the distance, we must choose the minus sign in Eq. (21). The right-hand side of (21) can be expressed in terms of $\xi$ and $\xi^{\prime}$, and then provides the squared distance between $\xi$ and $\xi^{\prime}$,

$$
d_{M}\left(\xi, \xi^{\prime}\right)^{2}=r+r^{\prime}-\sqrt{2} \sqrt{r r^{\prime}+\sum_{k=1}^{3} \xi_{k} \xi_{k}^{\prime}},
$$

where

$$
r=\sqrt{\sum_{k=1}^{3} \xi_{k}^{2}}, \quad r^{\prime}=\sqrt{\sum_{k=1}^{3} \xi_{k}^{\prime 2}}
$$

\section{EXCHANGES OF PARTICLES}

In Sec. III, we made use of the rotational symmetry to obtain the shape space $M$. We now turn to another symmetry, the symmetry of configurations arising from exchanges of identical particles. Thus, in this section, we assume that all particles are identical, and without loss of generality put $m_{j}=1, j=1,2,3$. Then the Jacobi vectors defined in Eq. (6) become

$$
\begin{gathered}
\mathbf{r}_{1}=\frac{1}{\sqrt{2}}\left(\mathbf{x}_{2}-\mathbf{x}_{1}\right), \\
\mathbf{r}_{2}=\sqrt{\frac{2}{3}}\left(\mathbf{x}_{3}-\frac{\mathbf{x}_{1}+\mathbf{x}_{2}}{2}\right) .
\end{gathered}
$$

Let $S_{3}$ be a symmetric group, the group of permutations of three symbols. If a configuration undergoes the change

$$
\left(\mathbf{x}_{1}, \mathbf{x}_{2}, \mathbf{x}_{3}\right) \mapsto\left(\mathbf{x}_{\sigma(1)}, \mathbf{x}_{\sigma(2)}, \mathbf{x}_{\sigma(3)}\right), \quad \sigma \in S_{3},
$$

the Jacobi vectors associated with the new configuration are given by

$$
\begin{gathered}
\mathbf{r}_{1}^{\sigma}=\frac{1}{\sqrt{2}}\left(\mathbf{x}_{\sigma(2)}-\mathbf{x}_{\sigma(1)}\right), \\
\mathbf{r}_{2}^{\sigma}=\sqrt{\frac{2}{3}}\left(\mathbf{x}_{\sigma(3)}-\frac{\mathbf{x}_{\sigma(1)}+\mathbf{x}_{\sigma(2)}}{2}\right) .
\end{gathered}
$$

The graphical representation of particle exchanges is given in Fig. 2, indicating which transformation takes the reference Jacobi vectors to which pair of new Jacobi vectors. From (26), one soon realizes that any particle exchanges can be represented by a linear transformation of Jacobi vectors $\mathbf{r}_{1}$ and $\mathbf{r}_{2}$. This will imply that the center-of-mass system of three identical particles admits the action of $S_{3}$ to the right.

We have to note here that since we are dealing with the right action of matrices, the representation of $S_{3}, \rho: S_{3} \rightarrow G L(2, \mathrm{R})$, must act on $X_{0}$ in the following manner:

$$
\left(\mathbf{r}_{1}, \mathbf{r}_{2}\right) \mapsto\left(\mathbf{r}_{1}^{h}, \mathbf{r}_{2}^{h}\right)=\left(\mathbf{r}_{1}, \mathbf{r}_{2}\right) \rho(h)^{-1}, \quad h \in S_{3}
$$

A straightforward calculation then provides 


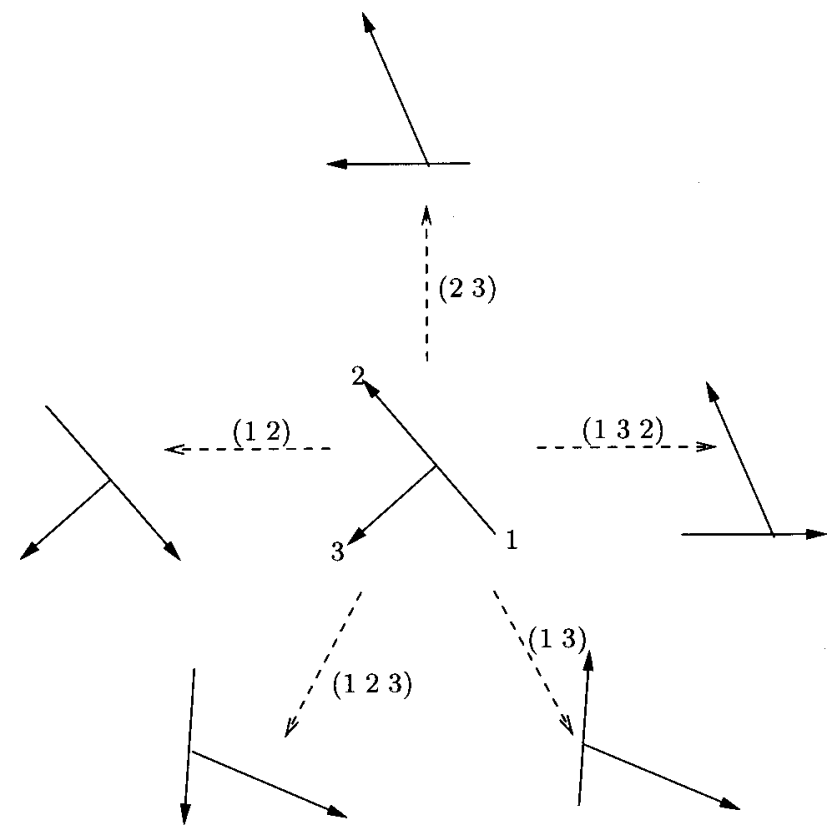

FIG. 2. This diagram represents the graphical view of all possible particle exchanges. Numbers in brackets are the elements of permutations from $S_{3}$. By the action of $\sigma \in S_{3}$, the site occupied by the particle $k$ in the reference configuration gets occupied in turn by the particle $\sigma(k)$.

$$
\begin{gathered}
\rho(e)=\left(\begin{array}{ll}
1 & 0 \\
0 & 1
\end{array}\right), \quad \rho\left(\begin{array}{ll}
1 & 2
\end{array}\right)=\left(\begin{array}{cc}
-1 & 0 \\
0 & 1
\end{array}\right), \\
\rho\left(\begin{array}{lll}
1 & 2 & 3
\end{array}\right)=\left(\begin{array}{cc}
-1 / 2 & \sqrt{3} / 2 \\
-\sqrt{3} / 2 & -1 / 2
\end{array}\right), \quad \rho\left(\begin{array}{lll}
1 & 3 & 2
\end{array}\right)=\left(\begin{array}{cc}
-1 / 2 & -\sqrt{3} / 2 \\
\sqrt{3} / 2 & -1 / 2
\end{array}\right), \\
\rho\left(\begin{array}{ll}
2 & 3
\end{array}\right)=\left(\begin{array}{cc}
1 / 2 & \sqrt{3} / 2 \\
\sqrt{3} / 2 & -1 / 2
\end{array}\right), \quad \rho\left(\begin{array}{ll}
1 & 3
\end{array}\right)=\left(\begin{array}{cc}
1 / 2 & -\sqrt{3} / 2 \\
-\sqrt{3} / 2 & -1 / 2
\end{array}\right) .
\end{gathered}
$$

It is an easy matter to verify that the matrices in (28) form a discrete subgroup of $\mathrm{O}(2)$ which is isomorphic to the symmetric group $S_{3}$. It is also well known that the set of the matrices given in (28) forms a unitary irreducible representation of $S_{3} .{ }^{15}$

So far we have not touched upon collision of particles, or excluded collision configurations from the center-of-mass system $X_{0}$. A remarkable point to make on the $S_{3}$ action on $X_{0}$ is that $S_{3}$ acts on the whole space $X_{0}$. This means that even if two or three particles collide, where the rank of the $2 \times 2$ matrix $\left(\mathbf{r}_{1}, \mathbf{r}_{2}\right)$ is less than two, Eq. (27) is applicable together with (28).

\section{THE ACTION OF $S_{3}$ ON $M$}

In Sec. IV, we have observed that the exchanges of identical particles give rise to the action of $S_{3}$ on $X_{0}$. With the identification $\dot{X}_{0} \cong \dot{\mathrm{C}}^{2}$, the action of $S_{3}$ on $\dot{X}_{0}$ is expressed, like (27), as

$$
\left(z_{1}, z_{2}\right) \mapsto\left(z_{1}, z_{2}\right) \rho(h)^{-1}, \quad h \in S_{3} .
$$

Since the action of $\mathrm{SO}(2)$ and of $S_{3}$ on $X_{0}$ commute, the action of $S_{3}$ on $M$ can be defined through 


$$
[x] \tau(h)^{-1}:=\left[x \rho(h)^{-1}\right], \quad x \in \dot{X}_{0} \cong \dot{C}^{2}, \quad h \in S_{3},
$$

where $\tau$ is a homomorphism: $S_{3} \rightarrow \mathrm{GL}(3, \mathrm{R})$, and represented as

$$
\left(\xi_{1}, \xi_{2}, \xi_{3}\right) \mapsto\left(\xi_{1}, \xi_{2}, \xi_{3}\right) \tau(h)^{-1}, \quad\left(\xi_{1}, \xi_{2}, \xi_{3}\right) \in M, \quad h \in S_{3} .
$$

Note here that the definition (30) is independent of the choice of representatives. A straightforward calculation along with (12), (28), and (30) shows that $\tau\left(S_{3}\right)$ forms a discrete subgroup of $\mathrm{SO}(3)$, which is expressed as

$$
\begin{gathered}
\tau(e)=\left(\begin{array}{lll}
1 & 0 & 0 \\
0 & 1 & 0 \\
0 & 0 & 1
\end{array}\right), \quad \tau\left(\begin{array}{ll}
1 & 2
\end{array}\right)=\left(\begin{array}{ccc}
-1 & 0 & 0 \\
0 & -1 & 0 \\
0 & 0 & 1
\end{array}\right), \\
\tau\left(\begin{array}{lll}
1 & 2 & 3
\end{array}\right)=\left(\begin{array}{ccc}
-1 / 2 & 0 & -\sqrt{3} / 2 \\
0 & 1 & 0 \\
\sqrt{3} / 2 & 0 & -1 / 2
\end{array}\right), \quad \tau\left(\begin{array}{lll}
1 & 3 & 2
\end{array}\right)=\left(\begin{array}{ccc}
-1 / 2 & 0 & \sqrt{3} / 2 \\
0 & 1 & 0 \\
-\sqrt{3} / 2 & 0 & -1 / 2
\end{array}\right), \\
\tau\left(\begin{array}{ll}
2 & 3
\end{array}\right)=\left(\begin{array}{ccc}
1 / 2 & 0 & \sqrt{3} / 2 \\
0 & -1 & 0 \\
\sqrt{3} / 2 & 0 & -1 / 2
\end{array}\right), \quad \tau\left(\begin{array}{ll}
1 & 3
\end{array}\right)=\left(\begin{array}{ccc}
1 / 2 & 0 & -\sqrt{3} / 2 \\
0 & -1 & 0 \\
-\sqrt{3} / 2 & 0 & -1 / 2
\end{array}\right) .
\end{gathered}
$$

At first sight, the dimension of matrices presented in (32) is $3 \times 3$, which is larger than those presented in (28), resulting in an increase in the number of dimension by one. This seems not to fit the fact that the dimension of $M$ is less than that of $\dot{X}_{0}$. However, this is not a contradiction. While we have identified $X_{0}$ with $\mathrm{R}^{2 \times 2}$, the set of Jacobi vectors, we are allowed alternatively to identify $X_{0}$ with $\mathrm{R}^{4}$, the set of row vectors of length 4 , so that we would have seen a discrete subgroup of GL(4,R) acting on $\mathrm{R}^{4}$, and would have been able to see an immediate reduction in the size of matrices. In fact, the $\mathrm{O}(2)$ action given in (27) proves to take the form

$$
\left(q_{1}, q_{2}, q_{3}, q_{4}\right) \mapsto\left(q_{1}, q_{2}, q_{3}, q_{4}\right)\left(\begin{array}{cc}
a I_{2} & b I_{2} \\
c I_{2} & d I_{2}
\end{array}\right)^{-1} \quad \text { for } \quad \rho(h)=\left(\begin{array}{ll}
a & b \\
c & d
\end{array}\right),
$$

where $h \in S_{3}$ and $I_{2}$ denotes the $2 \times 2$ unit matrix. We also see that the determinant of the $4 \times 4$

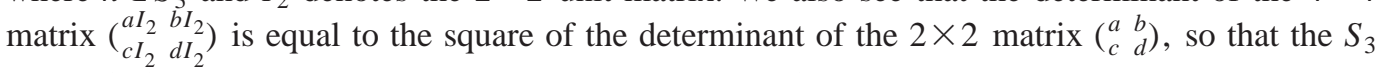
action (33) is represented as a discrete subgroup of $\mathrm{SO}(4)$.

\section{REDUCTION BY ROTATIONAL SYMMETRY}

In this section, we present the reduction of a quantum system on the center-of-mass system $X_{0}$ by rotational symmetry. The reduction procedure runs irrespectively of whether all particles are identical or not. We first need a decomposition of $L^{2}\left(C^{2}\right)$ with respect to the $\mathrm{SO}(2)$ action. For $f \in L^{2}\left(\mathrm{C}^{2}\right)$ given, we consider a function $f\left(e^{i s} z\right)$ with a parameter $s$, which can be expanded into the Fourier series

$$
f\left(e^{i s} z\right)=\sum_{m=-\infty}^{\infty} f_{m}(z) e^{i m s}, \quad f_{m}(z)=\frac{1}{2 \pi} \int_{-\pi}^{\pi} f\left(e^{i s} z\right) e^{-i m s} \mathrm{~d} s .
$$

In particular, for $s=0$, one has

$$
f(z)=\sum_{m=-\infty}^{\infty} f_{m}(z)
$$


Note here that the function $f_{m}$ defined in (34) satisfies $f_{m}\left(e^{i s} z\right)=e^{i m s} f_{m}(z)$. In general, a function $F$ on $\mathrm{C}^{2}$ satisfying the condition

$$
F\left(e^{i s} z\right)=e^{i m s} F(z)
$$

is called $\rho_{m}$-equivariant, where $\rho_{m}$ denotes a unitary irreducible representation of $\mathrm{SO}(2)$ $\cong \mathrm{U}(1) ; \rho_{m}\left(e^{i s}\right)=e^{i m s}$ with $m \in \mathrm{Z}$. As is easily seen, the decomposition (35) has remarkable properties,

$$
\|f\|^{2}=\sum_{m=-\infty}^{\infty}\left\|f_{m}\right\|^{2}, \quad\left\langle f_{n}, f_{m}\right\rangle=0 \quad \text { if } \quad n \neq m
$$

Thus $L^{2}\left(\mathrm{C}^{2}\right)$ is decomposed into the orthogonal direct sum,

$$
L^{2}\left(C^{2}\right)=\bigoplus_{m=-\infty}^{\infty} L_{m}^{2}\left(C^{2}\right)
$$

where each $L_{m}^{2}\left(C^{2}\right)$ is the space of equivariant functions,

$$
L_{m}^{2}\left(\mathrm{C}^{2}\right)=\left\{f \in L^{2}\left(\mathrm{C}^{2}\right) \mid f\left(e^{i s} z\right)=e^{i m s} f(z)\right\} .
$$

Suppose we are given a quantum dynamical system on $L^{2}\left(\mathrm{C}^{2}\right)$, of which the time evolution is expressed as a unitary transformation,

$$
\psi_{t}(z)=\int_{\mathrm{C}^{2}} G_{t}(z, w) \psi_{t_{0}}(w) \mathrm{d} w
$$

where $G_{t}(z, w)$ is a Green's function. Moreover, we assume that this quantum system is $\mathrm{U}(1)$ invariant, so that $G_{t}(z, w)$ is required to be $\mathrm{U}(1)$ invariant,

$$
G_{t}\left(e^{i s} z, e^{i s} w\right)=G_{t}(z, w) .
$$

Our task in the following is to decompose the time evolution (40) in $L^{2}\left(\mathrm{C}^{2}\right)$ into a series of those in respective subspaces $L_{m}^{2}\left(\mathrm{C}^{2}\right)$. This process will be called the reduction of the quantum system for simplicity. We will see later how the time evolution in $L_{m}^{2}\left(\mathrm{C}^{2}\right)$ is looked upon as the time evolution of a state on the internal space $M$. Since the Lebesgue measure $\mathrm{d} w$ on $\mathrm{C}^{2}$ is invariant under the $\mathrm{U}(1)$ action, and since $\psi_{t_{0}}\left(e^{i s} w\right)$ can be expanded into a Fourier series $\Sigma \psi_{t_{0}}^{m}(w) e^{i m s}$, the time evolution (40) can be decomposed into the following series:

$$
\begin{aligned}
\psi_{t}(z) & =\frac{1}{2 \pi} \int_{-\pi}^{\pi} \mathrm{d} s \int_{\mathrm{C}^{2}} G_{t}(z, w) \psi_{t_{0}}(w) \mathrm{d} w \\
& =\frac{1}{2 \pi} \int_{-\pi}^{\pi} \mathrm{d} s \int_{\mathrm{C}^{2}} G_{t}\left(z, e^{-i s} w\right) \psi_{t_{0}}\left(e^{-i s} w\right) \mathrm{d} w \\
& =\sum_{m=-\infty}^{\infty} \int_{\mathrm{C}^{2}} G_{t}^{m}(z, w) \psi_{t_{0}}^{m}(w) \mathrm{d} w
\end{aligned}
$$

where

$$
G_{t}^{m}(z, w):=\frac{1}{2 \pi} \int_{-\pi}^{\pi} G_{t}\left(e^{i s} z, w\right) e^{-i m s} \mathrm{~d} s
$$

and we have assumed that the order of integration and summation can be interchanged safely, which is the case for $\psi_{t_{0}} \in \mathcal{S}\left(\mathrm{C}^{2}\right)$, rapidly decreasing $C^{\infty}$ functions. At a glance of the definition of $G_{t}^{m}(z, w)$, we can observe that 


$$
\begin{gathered}
G_{t}^{m}\left(e^{i s} z, w\right)=e^{i m s} G_{t}^{m}(z, w), \\
G_{t}^{m}\left(z, e^{i s} w\right)=G_{t}^{m}(z, w) e^{-i m s} .
\end{gathered}
$$

On account of (44), the integral transform

$$
\psi_{t}^{m}(z)=\int_{\mathrm{C}^{2}} G_{t}^{m}(z, w) \psi_{t_{0}}^{m}(w) \mathrm{d} w
$$

proves to be in $L_{m}^{2}\left(\mathrm{C}^{2}\right)$, so that the map $\psi_{t_{0}}^{m} \mapsto \psi_{t}^{m}$ becomes also unitary. Thus we have decomposed the time evolution $\psi_{t}$ of the original system in $L^{2}\left(\mathrm{C}^{2}\right)$ into a series of those $\psi_{t}^{m}$ in $L_{m}^{2}\left(\mathrm{C}^{2}\right)$,

$$
\psi_{t}(z)=\sum_{m=-\infty}^{\infty} \psi_{t}^{m}(z), \quad \psi_{t}^{m} \in L_{m}^{2}\left(\mathrm{C}^{2}\right)
$$

\section{BOUNDARY CONDITIONS}

In treating the shape space in Sec. III, we have started with the restricted center-of-mass system $\dot{X}_{0}$. In this section, we wish to take the whole space $X_{0}$ into account to consider what will happen when the three particles collide at a point. First we note that the quotient space of the whole space $X_{0}$ by the $\mathrm{SO}(2)$ action becomes homeomorphic to $\mathrm{R}^{3}$,

$$
X_{0} / \mathrm{SO}(2) \cong \dot{\mathrm{R}}^{3} \cup\{0\}=\mathrm{R}^{3}
$$

Let us be reminded of the fact that $M=\dot{X}_{0} / \mathrm{SO}(2) \cong \dot{\mathrm{R}}^{3}$ is made into a Riemannian manifold with metric $\widetilde{K}$. Since the motion of the three free particles is associated with a geodesic in $\dot{X}_{0}$, and since the three particles may collide at a point simultaneously, we may expect that the geodesics in $\dot{X}_{0}$ that correspond to collision motion may project to geodesics in $M$ which may get out of $M$ within a finite time. This suggests that the Riemannian manifold $(M, \widetilde{K})$ is not geodesically complete. To prove this, it suffices to show that there is a geodesics which gets to the origin of $\mathrm{R}^{3}$, a point out of $M=\dot{\mathrm{R}}^{3}$, within a finite time. One can indeed find such a geodesic as follows: Let $w, z \in \dot{\mathrm{C}}^{2}=\dot{X}_{0}$ such that $w=\lambda z$ with $\lambda \neq 1$ a real constant. Then the straight line $u(t)=w+t(z$ $-w)$, a geodesic in $\dot{X}_{0}$, proves to be horizontal, since Eq. (20) is satisfied by this $u(t)$. Now, it is clear that $u(t)$ projects to a geodesic $\pi(u(t))$ approaching the origin of $\mathrm{R}^{3}$. In fact, one has

$$
2 u_{1}(t) \overline{u_{2}(t)}=(\lambda+t(1-\lambda))^{2}\left(\xi_{1}+i \xi_{2}\right), \quad\left|u_{1}(t)\right|^{2}-\left|u_{2}(t)\right|^{2}=(\lambda+t(1-\lambda))^{2} \xi_{3},
$$

where $2 z_{1} \bar{z}_{2}=\xi_{1}+i \xi_{2},\left|z_{1}\right|^{2}-\left|z_{2}\right|^{2}=\xi_{3}$, so that $\pi(u(t)) \rightarrow 0$ as $t \rightarrow \lambda /(\lambda-1)$. We have to point out in addition that Eq. (49) allows of the interpretation that the geodesic $\pi(u(t))$ remains to exist after getting out of $M$ for an instant. In fact, we may interpret that it traces backward the path it has printed before having reached the origin. This interpretation of the continuation of the geodesic comes from the mechanical fact that three particles on a plane may collide at a point, but they may continue to move after the collision.

We are to prove that if three particles collide at a point, the total angular momentum of them must vanish. Let $u(t)=w+t v$ be a geodesic in $\mathrm{C}^{2}$, which stands for a motion of free particles. We assume here that $w \neq 0, v \neq 0$. We are to consider whether or not $u(t)$ passes through the origin of $\mathrm{C}^{2}$. A necessary and sufficient condition for $|u(t)|^{2}=0$ for some real number $t$ is given by $(\operatorname{Re}\langle v, w\rangle)^{2}=|v|^{2}|w|^{2}$. But, in general, one has $|\langle v, w\rangle| \leqslant|v||w|$, so that

$$
\operatorname{Im}\langle v, w\rangle=0, \quad \text { and } \quad \lambda v+\mu w=0 \quad \text { for } \quad(\lambda, \mu) \neq 0, \lambda, \mu \in \mathrm{C} .
$$


Since $v \neq 0, w \neq 0$ by assumption, Eq. (50) implies that $v$ and $w$ are related by $v=k w$ with $k$ a nonzero real constant. Thus we have verified that if $|u(t)|=0$ for some $t, v$ and $w$ are related by $v=k w, k \in \mathrm{R}-\{0\}$. Moreover, the condition $\operatorname{Im}\langle v, w\rangle=0$ implies that

$$
\operatorname{Im}\left\langle u(t), \frac{\mathrm{d} u}{\mathrm{~d} t}\right\rangle=\operatorname{Im}\langle u(t), v\rangle=\operatorname{Im}\langle w, v\rangle=0
$$

which means that the angular momentum of the orbit $u(t)$ must vanish [see (20)]. Thus we conclude that if three free particles collide at a point simultaneously, the total angular momentum of them must vanish. By contraposition, three free particles with nonvanishing total angular momentum do not collide simultaneously at a point.

This observation suggests that we consider what happens in wave functions at $z=0$ according to whether the total angular momentum vanishes or not. We have to note here that the $L_{m}^{2}\left(\mathrm{C}^{2}\right)$ is the space of wave functions with the total angular momentum $m$. In fact, the $\rho_{m}$-equivariance condition (36) for a smooth function $F$ is differentiated with respect to $s$ at $s=0$ to provide

$$
\frac{1}{i}\left(-q_{2} \frac{\partial}{\partial q_{1}}+q_{1} \frac{\partial}{\partial q_{2}}-q_{4} \frac{\partial}{\partial q_{3}}+q_{3} \frac{\partial}{\partial q_{4}}\right) F(z)=m F(z)
$$

where the operator on the left-hand side stands for the total angular momentum operator. Moreover, condition (36) implies that $F(0)=e^{i m s} F(0)$ for $z=0$, and hence

$$
F(0)=0 \text { for } m \neq 0,
$$

which means that if the total angular momentum does not vanish $(m \neq 0)$, the three particles do not collide at the origin. If $m=0$, then $F(0)$ should be a finite value.

If some of the wave functions are analytic at $z=0$, we can describe more of the behavior of them at $z=0$. Let $f(z)$ be a function analytic at $z=0$. Then it may be expanded into a power series such as $f(z)=\sum c_{j_{1} j_{2} k_{1} k_{2}} z_{1}^{j_{1}} z_{2}^{j_{2}} z_{1}^{k_{1}} z_{2}^{k_{2}}$. We can rewrite this series in the form

$$
f(z)=\sum_{m=0}^{\infty} \sum_{n=m}^{\infty} \sum_{\substack{j_{1}+j_{2}-k_{1}-k_{2}=m \\ j_{1}+j_{2}+k_{1}+k_{2}=n}} c_{j_{1} j_{2} k_{1} k_{2}} z_{1}^{j_{1}} z_{2}^{j_{2}} z_{1}^{k_{1}} z_{2}^{k_{2}}+\sum_{m=-1}^{-\infty} \sum_{n=|m|}^{\infty} \sum_{\substack{j_{1}+j_{2}-k_{1}-k_{2}=m \\ j_{1}+j_{2}+k_{1}+k_{2}=n}} *
$$

This expansion shows that $f(z)$ is broken up into a series of $\rho_{m}$-equivariant functions, each of which takes the form of power series starting with a term of order $|m|$ irrespective of whether $m$ is non-negative or negative. Further, since $n=m+2\left(k_{1}+k_{2}\right)$, the power series starting with a term of order $|m|$ contains only terms of every other higher order. This fact was pointed out for planar two-body systems, ${ }^{16}$ while Ref. 16 mainly studies the behavior of wave functions at the collinear configurations of spatial three-body systems.

In the rest of this section, we describe analytic $\rho_{m}$-equivariant functions in terms of the local coordinate system $(R, \theta, \phi, \psi)$ introduced through

$$
\begin{gathered}
z_{1}=\operatorname{Re}^{i(\psi+\phi) / 2} \cos \frac{\theta}{2}, \quad z_{2}=\operatorname{Re}^{i(\psi-\phi) / 2} \sin \frac{\theta}{2}, \\
R \geqslant 0, \quad 0 \leqslant \theta \leqslant \pi, \quad 0 \leqslant \psi \leqslant 4 \pi, \quad 0 \leqslant \phi \leqslant 2 \pi .
\end{gathered}
$$

A $\rho_{m}$-equivariant function $F$ which is assumed to be analytic at $z=0$ is then expanded, on account of (54), into a power series of the form

$$
F(z)=e^{i m \psi / 2} \sum_{\ell=0}^{\infty} R^{|m|+2 \ell} \Phi_{m \ell}(\theta, \phi)
$$


where

$$
\Phi_{m \ell}(\theta, \phi)=\sum_{\substack{j_{1}+j_{2}=1 / 2(|m|+m)+\ell \\ k_{1}+k_{2}=1 / 2(|m|-m)+\ell}} c_{j_{1} j_{2} k_{1} k_{2}} e^{i \phi\left(j_{1}-j_{2}-k_{1}+k_{2}\right) / 2}\left(\cos \frac{\theta}{2}\right)^{j_{1}+k_{1}}\left(\sin \frac{\theta}{2}\right)^{j_{2}+k_{2}} .
$$

From this, we observe that the $\rho_{m}$-equivariant function $F$ is expressed as $e^{i m \psi / 2}$ times a power series in $R$ which starts with a term of the lowest order $|m|$ and contains only terms of every other order. Since $R$ is a measure to describe how configurations of particles are distant from the triple collision, Eq. (57) implies that the more the total angular momentum $|m|$ grows, the less closely the particles get together.

\section{REDUCTION BY PARTICLE EXCHANGES}

This section deals with reduction due to exchanges of identical particles. According to whether particles are all bosons or fermions, the wave function must be symmetric or antisymmetric with respect to particle exchanges. From a wave function $\psi$ on the center-of-mass system $X_{0} \cong \mathrm{C}^{2}$, we can construct such wave functions $\psi^{(s)}$ and $\psi^{(a)}$ by the following procedure;

$$
\begin{gathered}
\psi^{(s)}(x):=\sum_{h \in S_{3}} \psi\left(x \rho(h)^{-1}\right), \\
\psi^{(a)}(x):=\sum_{h \in S_{3}} \operatorname{sgn}(h) \psi\left(x \rho(h)^{-1}\right),
\end{gathered}
$$

where $\rho$ is the representation of $S_{3}$ in $\mathrm{O}(2)$, which is given in (28), and $\operatorname{sgn}(g)$ denotes the signum of $g$; $\operatorname{sgn}(g)$ equals 1 or -1 , depending on whether $g \in S_{3}$ is an even or odd permutation. It is easy to see that the $\psi^{(s)}$ and $\psi^{(a)}$ are symmetric and antisymmetric, respectively, with respect to the $S_{3}$ action,

$$
\psi^{(s)}\left(x \rho(g)^{-1}\right)=\psi^{(s)}(x), \quad \psi^{(a)}\left(x \rho(g)^{-1}\right)=\operatorname{sgn}(g) \psi^{(a)}(x) .
$$

In comparison with the $\rho_{m}$-equivariance with respect to the $\mathrm{SO}(2) \cong \mathrm{U}(1)$ action, Eq. (61) shows that bosonic and fermionic states are equivariant with respect to the trivial representation $\sigma \mapsto 1$ and to the signum representation $\sigma \mapsto \operatorname{sgn}(\sigma)$, respectively.

We assume here that our quantum system $\left(L^{2}\left(C^{2}\right), \psi_{t}\right)$ is invariant under the particle exchanges, so that $G_{t}(z, w)$ is assumed to be invariant under the action of $S_{3}$,

$$
G_{t}\left(z \rho(h)^{-1}, w \rho(h)^{-1}\right)=G_{t}(z, w), \quad h \in S_{3} .
$$

We note further that the Lebesgue measure $\mathrm{d} w$ on $X_{0} \cong \mathrm{R}^{4}$ is also invariant under the action of $S_{3}$. This is because the $S_{3}$ action on $X_{0}$ is represented as a discrete subgroup of $\mathrm{SO}(4)$, as is seen from (33). The invariance of the Green's kernel and of the measure $\mathrm{d} w$ under the $S_{3}$ action are put together to imply that the time evolution preserves the statistics to which the particles are subject, that is, bosonic or fermionic state remains unchanged during the time evolution. This can be seen from the change that the time evolution (40) undergoes by the $S_{3}$ action,

$$
\psi_{t}\left(z \rho(h)^{-1}\right)=\int_{\mathrm{C}^{2}} G_{t}(z, w) \psi_{t_{0}}\left(w \rho(h)^{-1}\right) \mathrm{d} w .
$$

Since the action of U(1) and of $S_{3}$ commute, the time evolution (63) is decomposed into

$$
\psi_{t}\left(z \rho(h)^{-1}\right)=\sum_{m=-\infty}^{\infty} \psi_{t}^{m}\left(z \rho(h)^{-1}\right)=\sum_{m=-\infty}^{\infty} \int_{\mathrm{C}^{2}} G_{t}^{m}(z, w) \psi_{t_{0}}^{m}\left(w \rho(h)^{-1}\right) \mathrm{d} w,
$$


where the Green's functions are invariant under the $S_{3}$ action,

$$
G_{t}^{m}\left(z \rho(h)^{-1}, w \rho(h)^{-1}\right)=G_{t}^{m}(z, w), \quad h \in S_{3} .
$$

Putting (64) together with (59) and (60), we obtain the time evolution of wave functions for Bose or Fermi particles, which are decomposed into

$$
\begin{gathered}
\psi_{t}^{(s)}(z)=\sum_{m=-\infty}^{\infty} \psi_{t}^{(s) m}(z), \quad \psi_{t}^{(s) m}(z):=\sum_{h \in S_{3}} \psi_{t}^{m}\left(z \rho(h)^{-1}\right), \\
\psi_{t}^{(a)}(z)=\sum_{m=-\infty}^{\infty} \psi_{t}^{(a) m}(z), \quad \psi_{t}^{(a) m}(z):=\sum_{h \in S_{3}} \operatorname{sgn}(h) \psi_{t}^{m}\left(z \rho(h)^{-1}\right),
\end{gathered}
$$

respectively.

Proposition 1: A planar three-particle quantum system $\left(L^{2}\left(\mathrm{C}^{2}\right), \psi_{t}\right)$ with symmetry of rotation and particle exchanges is reduced to subsystems $\left(L_{m}^{2}\left(\mathrm{C}^{2}\right), \psi_{t}^{(s) m}\right)$ or $\left(L_{m}^{2}\left(\mathrm{C}^{2}\right), \psi_{t}^{(a) m}\right)$ according to whether the particles are bosons or fermions, where $\psi_{t}^{(s) m}$ and $\psi_{t}^{(a) m}$ are given by (66) and (67), respectively, along with (46).

\section{COMPLEX LINE BUNDLES}

So far we have discussed the reduction to subsystems (see Proposition 1). In this section, we show that these subsystems indeed give rise to reduced quantum systems on the shape space, by introducing complex line bundles associated with the $\mathrm{U}(1)$ bundle $\dot{\mathrm{C}}^{2} \cong \dot{X}_{0} \rightarrow M$. To this end, we first recall that the time evolution $\psi_{t}$ in $L^{2}\left(\mathrm{C}^{2}\right)$ was decomposed into the series of those in $L_{m}^{2}\left(\mathrm{C}^{2}\right)$,

$$
\psi_{t}^{m}(z):=\int_{\mathrm{C}^{2}} G_{t}^{m}(z, w) \psi_{t_{0}}^{m}(w) \mathrm{d} w, \quad \psi_{t_{0}}^{m} \in L_{m}^{2}\left(\mathrm{C}^{2}\right) .
$$

Since the integrand in (68) is invariant under the $\mathrm{U}(1)$ action, the integration with respect to $w$ over $\mathrm{C}^{2}$ will reduce to that over the shape space $M$, if $\mathrm{C}^{2}$ is restricted to $\dot{\mathrm{C}}^{2}$. Hence the time evolution $\psi_{t}^{m}(z)$ may define the time evolution of a quantum state on the shape space $M$. In what follows, we make a brief review of complex line bundles associated with $\dot{\mathrm{C}}^{2} \cong \dot{X}_{0} \rightarrow M=\dot{\mathrm{R}}^{3}$ along with boundary conditions at the origin of $\mathrm{R}^{3}$.

For a unitary irreducible representation $\rho_{m}$, the complex line bundle $E_{m}$ associated with the $\mathrm{U}(1)$ bundle $\dot{X}_{0} \cong \dot{\mathrm{C}}^{2} \rightarrow M$ is defined to be the quotient of the product space $\dot{X}_{0} \times \mathrm{C}$ by the equivalence relation defined through $(z, \zeta) \sim\left(e^{i s} z, e^{i m s} \zeta\right)$ for $(z, \zeta) \in \dot{\mathrm{C}}^{2} \times \mathrm{C}$. By $[(z, \zeta)]$ and by $\pi_{m}$ we denote the equivalence class in $\dot{X}_{0} \times \mathrm{C}$ and the projection $E_{m} \rightarrow M$, respectively, so that one has $\pi_{m}([(z, \zeta)])=\pi(z)$. A section $\sigma$ in $E_{m}$ is a map $M \rightarrow E_{m}$ such that $\pi_{m}{ }^{\circ} \sigma=\mathrm{id}_{M}$, where $\operatorname{id}_{M}$ is the identity map of $M$. Then any $\rho_{m}$-equivariant function $F$ on $\dot{X}_{0}$ determines a section $\sigma$ in $E_{m}$ by

$$
\sigma(\pi(z))=[(z, F(z))] .
$$

Sections in $E_{m}$ and $\rho_{m}$-equivariant functions are in one-to-one correspondence. Furthermore, from (53), $\rho_{m}$-equivariant functions should be subject to the boundary condition that $F(z) \rightarrow 0$ as $z$ $\rightarrow 0$ if $m \neq 0$ and that $F(z)$ is bounded as $z \rightarrow 0$ if $m=0$, so that the corresponding section $\sigma(\xi)$ should satisfy the corresponding boundary condition as $\xi \rightarrow 0$ in $M$.

For sections $\sigma_{1}$ and $\sigma_{2}$ corresponding to $\rho_{m}$-equivariant functions $F_{1}$ and $F_{2}$, respectively, the inner product $\left\langle\sigma_{1}, \sigma_{2}\right\rangle$ is defined to be

$$
\left\langle\sigma_{1}, \sigma_{2}\right\rangle=\int_{M}\left(\sigma_{1}, \sigma_{2}\right) \mathrm{d} \mu_{M}=\int_{\mathrm{C}^{2}} \overline{F_{1}(z)} F_{2}(z) \mathrm{d} z,
$$


where $\left(\sigma_{1}, \sigma_{2}\right)$ denotes the inner product in each fiber $\pi_{m}^{-1}(\pi(z)) \cong \mathrm{C}$, and $\mathrm{d} \mu_{M}$ is the measure on $M$ defined for any function $\chi$ on $M$ through

$$
\int_{M} \chi(p) \mathrm{d} \mu_{M}=\int_{C^{2}} \chi(\pi(z)) \mathrm{d} z \quad \text { with } \quad \pi(z)=p \in M .
$$

To put the measure $\mathrm{d} \mu_{M}$ in an explicit form, it is of great help to use the connection form $\omega$ given in (15). A straightforward calculation then shows that the standard volume form on $X_{0}$ is put in the form

$$
d q_{1} \wedge d q_{2} \wedge d q_{3} \wedge d q_{4}=\frac{1}{8 r} \omega \wedge d \xi_{1} \wedge d \xi_{2} \wedge d \xi_{3}, \quad r=\sqrt{\xi_{1}^{2}+\xi_{2}^{2}+\xi_{3}^{2}}
$$

where $\xi_{k}, k=1,2,3$, are defined in (12). To be precise, $d \xi_{k}$ on the right-hand side of (72) should be replaced by $\pi^{*} d \xi_{k}$, the pull-back of $d \xi_{k}$ through $\pi$, but we have used $d \xi_{k}$ for simplicity. From (71) and (72), we conclude after the integration over the fiber $S^{1} \cong \mathrm{U}(1)$ that

$$
\mathrm{d} \mu_{M}=\frac{\pi}{4 r} d \xi_{1} \wedge d \xi_{2} \wedge d \xi_{3}
$$

From (69) and (70), we see that any function $F \in L_{m}^{2}\left(\dot{\mathrm{C}}^{2}\right)$ determines a square integrable section in $E_{m}$. Taking into account the above-mentioned boundary condition for $\rho_{m}$-equivariant functions, we may regard $L_{m}^{2}\left(C^{2}\right)$ as being in one-to-one correspondence to the space of square integrable sections in $E_{m}$ together with the boundary condition.

For the $\rho_{m}$-equivariant function $\psi_{t}^{m}(z)$ given in (68), one has the time evolution of the corresponding section $\sigma_{t}^{m}$ in $E_{m}$ together with the boundary condition,

$$
\sigma_{t}^{m}(\pi(z))=\left[\left(z, \psi_{t}^{m}(z)\right)\right] .
$$

Since the time evolution $\psi_{t}^{m}$ is unitary, that is, $\left\|\psi_{t}^{m}\right\|=\left\|\psi_{t_{0}}^{m}\right\|$, the time evolution of the corresponding section $\sigma_{t}^{m}$ is also unitary, that is, $\left\|\sigma_{t}^{m}\right\|=\left\|\sigma_{t_{0}}^{m}\right\|$ for all time $t$.

The $S_{3}$ action on $L_{m}^{2}\left(C^{2}\right)$ can be transferred to that on the space of square integrable sections in $E_{m}$. From (66) and (67), we obtain corresponding time evolutions of sections in $E_{m}$, respectively,

$$
\begin{gathered}
\sigma_{t}^{(s) m}(\pi(z)):=\sum_{h \in S_{3}} \sigma_{t}^{m}\left(\pi(z) \tau(h)^{-1}\right), \\
\sigma_{t}^{(a) m}(\pi(z)):=\sum_{h \in S_{3}} \operatorname{sgn}(h) \sigma_{t}^{m}\left(\pi(z) \tau(h)^{-1}\right) .
\end{gathered}
$$

The reduction is thus completed for the time evolution of a $\mathrm{U}(1) \cong \mathrm{SO}(2)$ invariant quantum system of three identical particles on a plane.

Theorem 2: If a quantum system for three identical particles on a plane admits the symmetry of rotation and particle exchanges, the time evolution of the quantum system, which is defined in the $L^{2}$ space of wave functions on the center-of-mass system, is reduced to that on the $L^{2}$ space of sections in the complex line bundle $E_{m}$ over the internal space $M$, where sections $\sigma$ must satisfy the boundary condition that $\sigma(\xi) \rightarrow 0$ as $\xi \rightarrow 0$ if $m \neq 0$ or that $\sigma(\xi)$ is bounded as $\xi \mapsto 0$ if $m$ $=0$. According to whether the quantum system is bosonic or fermionic, the time evolution is put in the form of (75) or (76). 


\section{APPLICATION TO FREE PARTICLES}

Having set up the reduction method, we are to apply it to a system of free particles. The Schrödinger equation for three free particles on a plane is expressed, in terms of $\left(q_{i}\right)$, as

$$
i \hbar \frac{\partial \psi}{\partial t}=-\frac{\hbar^{2}}{2} \nabla^{2} \psi \quad \text { with } \quad \nabla^{2}=\sum_{j=1}^{4} \frac{\partial^{2}}{\partial q_{j}^{2}} .
$$

In fact, since the operator $\Sigma_{k=1}^{3}\left(1 / m_{k}\right)\left(\partial / \partial \mathbf{x}_{k}\right)^{2}$, a constant multiple of the kinetic energy operator, is the Laplacian $\nabla^{2}$ with respect to the metric (1) on $X$, and since this metric is expressed as $\Sigma_{j=1}^{4} d q_{j}^{2}$ if restricted to the linear subspace $X_{0}$ of $X$, the Laplacian takes the form $\nabla^{2}$ $=\sum_{j=1}^{4} \partial^{2} / \partial q_{j}^{2}$. As is well known, Eq. (77) can be solved by Fourier transform with little difficulty, to give a solution of the form

$$
\psi_{t}(z)=\int_{\mathrm{C}^{2}} F_{t}(z, w) \psi_{t_{0}}(w) \mathrm{d} w
$$

where $F_{t}$ is the Green's function given by

$$
F_{t}(z, w)=\left[\frac{1}{2 \pi i \hbar\left(t-t_{0}\right)}\right]^{2} \exp \left(\frac{i|z-w|^{2}}{2 \hbar\left(t-t_{0}\right)}\right) .
$$

Since the Schrödinger equation (77) is invariant under the $U(1)$ action (10), the free particle system can be reduced after the procedure in Sec. VI. As is easily seen from (79), the Green's kernel $F_{t}(z, w)$ satisfies the condition (41), so that we can compute explicitly Eq. (43) with $F_{t}$ replaced for $G_{t}$ to obtain

$$
F_{t}^{m}(z, w)=\frac{e^{i m(\theta(z, w)-\pi / 2)}}{\left(2 \pi i \hbar\left(t-t_{0}\right)\right)^{2}} \exp \left(\frac{i B(z, w)}{2 \hbar\left(t-t_{0}\right)}\right) J_{m}\left(\frac{A(z, w)}{\hbar\left(t-t_{0}\right)}\right),
$$

where $J_{m}$ is the Bessel function defined as

$$
J_{m}(x)=\frac{1}{2 \pi} \int_{-\pi}^{\pi} e^{i x \sin s} e^{-i m s} \mathrm{~d} s, \quad x \in \mathrm{C},
$$

and

$$
\begin{gathered}
B(z, w)=\sum_{j=1}^{2}\left(\left|z_{j}\right|^{2}+\left|w_{j}\right|^{2}\right), \\
A(z, w)=\left|\sum_{j=1}^{2} z_{j} \bar{w}_{j}\right|, \\
\theta(z, w)=\arg \sum_{j=1}^{2} z_{j} \bar{w}_{j} .
\end{gathered}
$$

It is of great interest to observe that $A(z, w)$ and $B(z, w)$ are invariant under the $\mathrm{U}(1)$ action, $z \mapsto e^{i s} z$ and/or $w \mapsto e^{i s} w$, and hence can be expressed in the coordinates of the shape space $M$. In fact, we can verify that

$$
B(z, w)=\widetilde{B}\left(\xi, \xi^{\prime}\right):=\sqrt{\sum_{k=1}^{3} \xi_{k}^{2}}+\sqrt{\sum_{k=1}^{3} \xi_{k}^{\prime 2}},
$$




$$
A(z, w)=\tilde{A}\left(\xi, \xi^{\prime}\right):=\left[\frac{1}{2} \sqrt{\sum_{k=1}^{3} \xi_{k}^{2}} \sqrt{\sum_{k=1}^{3} \xi_{k}^{\prime 2}}+\frac{1}{2} \sum_{k=1}^{3} \xi_{k} \xi_{k}^{\prime}\right]^{1 / 2},
$$

where $\xi_{k}^{\prime}$ are given by the formula similar to (12). We notice further that under the U(1) action the factor $e^{i m \theta(z, w)}$ is subject to the transformation

$$
\begin{aligned}
& \exp \left(i m \theta\left(e^{i s} z, w\right)\right)=e^{i m s} \exp (i m \theta(z, w)), \\
& \exp \left(i m \theta\left(z, e^{i s} w\right)\right)=\exp (i m \theta(z, w)) e^{-i m s} .
\end{aligned}
$$

Properties (85)-(87) show that $F_{t}^{m}$ is subject to the transformations (44) and (45). Thus the time evolution (78) is reduced to (46) with $G_{t}^{m}$ replaced by $F_{t}^{m}$.

Furthermore, since $F_{t}$ is invariant under the $S_{3}$ action, as is seen from (79), so is $F_{t}^{m}$. Thus the free particle system reduces to subsystems according to Proposition 1. Finally, application of Theorem 2 provides reduced systems defined on the complex line bundles $E_{m}$.

In conclusion, we note that the reduced Hamiltonian operator, which acts on sections of $E_{m}$ and is denoted by $\hat{H}_{m}$, is given by

$$
\hat{H}_{m}=-\frac{\hbar^{2}}{2} 4 r \sum_{k=1}^{3} \nabla_{k}^{2}+\frac{m^{2}}{2 r}
$$

where $\nabla_{k}$ 's are the covariant differential operators with respect to the vector fields $\partial / \partial \xi_{k}$. See Ref. 1 for details, in which the reduced Hamiltonian operator was studied for a generic Hamiltonian system of planar three particles.

\section{LOCAL EXPRESSION}

The purpose of this section is to look into the boundary conditions and the Green's function discussed in previous sections, in terms of local coordinates.

We first consider the boundary condition at $z=0$ for $\rho_{m}$-equivariant functions in the coordinates $(R, \theta, \phi, \psi)$ introduced in (55). As is easily seen from (12) and (55), one has

$$
\xi_{1}+i \xi_{2}=r e^{i \phi} \sin \theta, \quad \xi_{3}=r \cos \theta, \quad r=R^{2}
$$

which means that $(r, \theta, \phi)$ serve as spherical polar coordinates in the shape space $M \cong \dot{\mathrm{R}}^{3}$. We take a local section, $s=\left(s_{1}, s_{2}\right)$, in the bundle $\dot{X}_{0} \cong \dot{\mathrm{C}}^{2} \rightarrow M$ as follows:

$$
s_{1}(r, \theta, \phi)=\sqrt{r} e^{i \phi / 2} \cos \frac{\theta}{2}, \quad s_{2}(r, \theta, \phi)=\sqrt{r} e^{-i \phi / 2} \sin \frac{\theta}{2},
$$

where

$$
r>0, \quad 0<\theta<\pi, \quad 0<\phi<2 \pi
$$

Then one has $z=e^{i \psi / 2} s(r, \theta, \phi)$, so that the local section corresponding to a $\rho_{m}$-equivariant function $F$ is expressed as $[(z, F(z))]=[s(r, \theta, \phi), F \circ s(r, \theta, \phi)]$. This implies that $F \circ s$ may be identified with a local section in $E_{m}$. If $F$ is given by (57), we obtain

$$
F \circ s(r, \theta, \phi)=r^{|m| / 2} \sum_{\ell=0}^{\infty} r^{\ell} \Phi_{m \ell}(\theta, \phi)
$$


This shows that if a $\rho_{m}$-equivariant function $F(z)$ is analytic in $R$ at $R=0$, then the corresponding local section is expressed as $r^{|m| / 2}$ times an analytic function in $r$. In particular, the local section associated with the kernel function $F_{t}^{m}$, as a function of $z$, given in (80) can be expanded in this form.

We turn to expressing the integral transform (68) with $G_{t}^{m}$ replaced by $F_{t}^{m}$ as the integral of a function on the shape space $M$ explicitly. To this end, we use local sections $\sigma_{+}$and $\sigma_{-}$in the bundle $\dot{X}_{0} \rightarrow M$, which are defined to be

$$
\begin{aligned}
& \sigma_{+}(\xi)=\left(\frac{\sqrt{r+\xi_{3}}}{\sqrt{2}}, \frac{\xi_{1}-i \xi_{2}}{\sqrt{2\left(r+\xi_{3}\right)}}\right) \quad \text { for } \xi \in D_{+}, \\
& \sigma_{-}(\xi)=\left(\frac{\xi_{1}+i \xi_{2}}{\sqrt{2\left(r-\xi_{3}\right)}}, \frac{\sqrt{r-\xi_{3}}}{\sqrt{2}}\right) \quad \text { for } \xi \in D_{-},
\end{aligned}
$$

where $D_{ \pm}$are domains in $M$ defined, respectively, to be

$$
\begin{aligned}
& D_{+}=\left\{\xi \in \dot{\mathrm{R}}^{3} \mid \xi_{3}+r \neq 0\right\}, \\
& D_{-}=\left\{\xi \in \dot{\mathrm{R}}^{3} \mid \xi_{3}-r \neq 0\right\} .
\end{aligned}
$$

The sections $\sigma_{+}$and $\sigma_{-}$are subject to the transformation in the intersection $D_{+} \cap D_{-}$,

$$
\sigma_{-}(\xi)=\frac{\xi_{1}+i \xi_{2}}{\sqrt{\xi_{1}^{2}+\xi_{2}^{2}}} \sigma_{+}(\xi), \quad \xi \in D_{+} \cap D_{-} .
$$

By using the section $\sigma_{+}$, points of $\pi^{-1}\left(D_{+}\right)$are expressed as $z=e^{i \varphi} \sigma_{+}(\xi)$ with $\xi=\pi(z)$ and $\varphi$ an angle variable. Thus local coordinates $(\xi, \varphi)$ are introduced in $\pi^{-1}\left(D_{+}\right)$. Local coordinates are defined in $\pi^{-1}\left(D_{-}\right)$as well. Then, a $\rho_{m}$-equivariant function $F$ restricted on $\pi^{-1}\left(D_{+}\right)$is put in the form $F(z)=e^{i m \varphi} F\left(\sigma_{+}(\xi)\right)$. A similar expression of $F$ is available on $\pi^{-1}\left(D_{+}\right)$.

We first divide $M$ into a union $M=M_{+} \cup M_{-}$, where $M_{ \pm}$are the upper and the lower half spaces of $M \cong \dot{\mathrm{R}}^{3} ; M_{+}=\left\{\xi \in M \mid \xi_{3} \geqslant 0\right\}, M_{-}=\left\{\xi \in M \mid \xi_{3} \leqslant 0\right\}$, and hence we have the division of $\dot{\mathrm{C}}^{2}$ in the form $\dot{\mathrm{C}}^{2}=\pi^{-1}\left(M_{+}\right) \cup \pi^{-1}\left(M_{-}\right)$. Accordingly, the integral transform (68) with $F_{t}^{m}$ replaced for $G_{t}^{m}$ is broken up into

$$
\psi_{t}^{m}(z)=\int_{\pi^{-1}\left(M_{+}\right)} F_{t}^{m}(z, w) \psi_{t_{0}}^{m}(w) \mathrm{d} w+\int_{\pi^{-1}\left(M_{-}\right)} *
$$

We now use the local sections $\sigma_{+}$and $\sigma_{-}$restricted on $M_{+}$and $M_{-}$, respectively, to rewrite the integrals (98) in terms of the variable $w=e^{i \varphi^{\prime}} \sigma_{+}\left(\xi^{\prime}\right) \in \pi^{-1}\left(M_{+}\right)$, etc. In particular, for $z$ $\in \pi^{-1}\left(D_{+}\right)$, Eq. (98) is put in the form

$$
\begin{aligned}
\psi_{t}^{m}\left(\sigma_{+}(\xi)\right)= & \int_{M_{+}} \widetilde{F}_{t}^{m}\left(\xi, \xi^{\prime}\right) e_{++}^{m}\left(\xi, \xi^{\prime}\right) \psi_{t_{0}}^{m}\left(\sigma_{+}\left(\xi^{\prime}\right)\right) \mathrm{d} \mu_{M}\left(\xi^{\prime}\right) \\
& +\int_{M_{-}} \widetilde{F}_{t}^{m}\left(\xi, \xi^{\prime}\right) e_{+-}^{m}\left(\xi, \xi^{\prime}\right) \psi_{t_{0}}^{m}\left(\sigma_{-}\left(\xi^{\prime}\right)\right) \mathrm{d} \mu_{M}\left(\xi^{\prime}\right),
\end{aligned}
$$

where the angle variables $\varphi$ has been canceled out from the both sides, and the definition (71) of $\mathrm{d} \mu_{M}$ has been used along with 


$$
\begin{aligned}
\widetilde{F}_{t}^{m}\left(\xi, \xi^{\prime}\right):= & \frac{e^{-i m \pi / 2}}{\left(2 \pi i \hbar\left(t-t_{0}\right)\right)^{2}} \exp \left(\frac{\widetilde{B}\left(\xi, \xi^{\prime}\right)}{2 \hbar\left(t-t_{0}\right)}\right) J_{m}\left(\frac{\widetilde{A}\left(\xi, \xi^{\prime}\right)}{\hbar\left(t-t_{0}\right)}\right), \\
& e_{++}^{m}\left(\xi, \xi^{\prime}\right):=e^{i m \theta\left(\sigma_{+}(\xi), \sigma_{+}\left(\xi^{\prime}\right)\right)}, \\
& e_{+-}^{m}\left(\xi, \xi^{\prime}\right):=e^{i m \theta\left(\sigma_{+}(\xi), \sigma_{-}\left(\xi^{\prime}\right)\right)} .
\end{aligned}
$$

A similar expression for $\psi_{t}^{m}\left(\sigma_{-}(\xi)\right), \xi \in D_{-}$, can be obtained as well with due definition of $e_{-+}^{m}\left(\xi, \xi^{\prime}\right)$ and of $e_{--}^{m}\left(\xi, \xi^{\prime}\right)$. The functions $\psi_{t}^{m}\left(\sigma_{-}(\xi)\right)$ and $\psi_{t}^{m}\left(\sigma_{+}(\xi)\right)$ are related on $D_{+} \cap D_{-}$ by

$$
\psi_{t}^{m}\left(\sigma_{-}(\xi)\right)=\left(\frac{\xi_{1}+i \xi_{2}}{\sqrt{\xi_{1}^{2}+\xi_{2}^{2}}}\right)^{m} \psi_{t}^{m}\left(\sigma_{+}(\xi)\right), \quad \xi \in D_{+} \cup D_{-},
$$

which is observed from (97) and from the fact that $\psi_{t}^{m}$ is $\rho_{m}$-equivariant.

In the rest of this section, we are to look into the function $\widetilde{F}_{t}^{m}\left(\xi, \xi^{\prime}\right)$ in detail by using asymptotic expansion of Bessel functions. As is well known, a simple form of the asymptotic expansion of $J_{n}(x)$ for $|x| \gg 1$ is given by

$$
\begin{aligned}
J_{m}(x) & \sim \sqrt{\frac{2}{\pi x}} \cos \left(x-\frac{1}{2} m \pi-\frac{1}{4} \pi\right) \\
& =\frac{1}{2} \sqrt{\frac{2}{\pi x}}\left(\exp \left(i\left(x-\frac{1}{2} m \pi-\frac{1}{4} \pi\right)\right)+\exp \left(-i\left(x-\frac{1}{2} m \pi-\frac{1}{4} \pi\right)\right)\right) .
\end{aligned}
$$

Then one has, for $0<\left|t-t_{0}\right| \hbar \ll \widetilde{A}\left(\xi, \xi^{\prime}\right)$,

$$
\begin{aligned}
J_{m}\left(\frac{\widetilde{A}\left(\xi, \xi^{\prime}\right)}{\left(t-t_{0}\right) \hbar}\right) & \sqrt{\frac{\left(t-t_{0}\right) \hbar}{2 \pi \widetilde{A}\left(\xi, \xi^{\prime}\right)}}\left(e^{-\pi i / 4} e^{-i m \pi / 2} \exp \left(i \frac{\widetilde{A}\left(\xi, \xi^{\prime}\right)}{\left(t-t_{0}\right) \hbar}\right)+e^{\pi i / 4} e^{i m \pi / 2}\right. \\
& \left.\times \exp \left(-i \frac{\widetilde{A}\left(\xi, \xi^{\prime}\right)}{\left(t-t_{0}\right) \hbar}\right)\right),
\end{aligned}
$$

where we have assumed that $\widetilde{A}\left(\xi, \xi^{\prime}\right) \neq 0$. We note in addition that $\widetilde{A}\left(\xi, \xi^{\prime}\right)=0$ with $\xi \neq 0, \xi^{\prime}$ $\neq 0$, if and only if there exists a positive constant $\lambda>0$ such that $\xi_{k}^{\prime}=-\lambda \xi_{k}, k=1,2,3$, as observed from (86). Inserting the asymptotic expansion (105) of $J_{m}\left(\widetilde{A}\left(\xi, \xi^{\prime}\right) /\left(t-t_{0}\right) \hbar\right)$ in the right-hand side of (100), one obtains, for $0<\left|t-t_{0}\right| \hbar \ll \widetilde{A}\left(\xi, \xi^{\prime}\right)$,

$$
\begin{aligned}
\widetilde{F}_{t}^{m}\left(\xi, \xi^{\prime}\right) \sim & -\frac{(-1)^{m} e^{-\pi i / 4}}{\widetilde{A}\left(\xi, \xi^{\prime}\right)^{1 / 2}} \frac{1}{\left(2 \pi\left(t-t_{0}\right) \hbar\right)^{3 / 2}} \exp \left(\frac{i}{2\left(t-t_{0}\right) \hbar}\left(r+r^{\prime}+2 \widetilde{A}\left(\xi, \xi^{\prime}\right)\right)\right) \\
& +\frac{1}{\widetilde{A}\left(\xi, \xi^{\prime}\right)^{1 / 2}} \frac{1}{\left(2 \pi i\left(t-t_{0}\right) \hbar\right)^{3 / 2}} \exp \left(\frac{i}{2\left(t-t_{0}\right) \hbar}\left(r+r^{\prime}-2 \widetilde{A}\left(\xi, \xi^{\prime}\right)\right)\right) .
\end{aligned}
$$

We now consider the quantity appearing in the argument of the exponential function in (106). To this end, let

$$
\alpha\left(\xi, \xi^{\prime}\right):=r+r^{\prime}+2 \widetilde{A}\left(\xi, \xi^{\prime}\right) .
$$


Then, differentiation of $\alpha$ with respect to $\xi^{\prime}$ implies that for nonvanishing $\xi^{\prime}, \partial \alpha / \partial \xi^{\prime}=0$ if and only if

$$
\sqrt{2} \sqrt{r r^{\prime}+\sum_{k=1}^{3} \xi_{k} \xi_{k}^{\prime}}+r+r^{\prime} \frac{\xi_{k}}{\xi_{k}^{\prime}}=0, \quad k=1,2,3,
$$

so that $\partial \alpha / \partial \xi_{k}^{\prime}=0$ if and only if $\xi_{k}=-\nu \xi_{k}^{\prime}, k=1,2,3$ for a positive constant $\nu>0$. Incidentally, for $\xi$ and $\xi^{\prime}$ with $\xi=-\nu \xi^{\prime}$, one obtains $\widetilde{A}\left(\xi, \xi^{\prime}\right)=0$, which contradicts the assumption that $\widetilde{A}\left(\xi, \xi^{\prime}\right) \neq 0$. From this it follows that if $\widetilde{A}\left(\xi, \xi^{\prime}\right) \neq 0$ then $\alpha\left(\xi, \xi^{\prime}\right)$ does not attain its stationary values, i.e., $\partial \alpha / \partial \xi_{k}^{\prime} \neq 0, k=1,2,3$, so that the function

$$
\exp \left(\frac{i}{2\left(t-t_{0}\right) \hbar} \alpha\left(\xi, \xi^{\prime}\right)\right)
$$

rapidly oscillates throughout the $\xi^{\prime}$-space with $\xi \neq-\nu \xi^{\prime}$. Hence, the first term (106) of the right-hand side of the asymptotic expansion (106) and (107) would make no contribution in the integral transform. As for the argument of the exponential function in the second term (107), we find that $r+r^{\prime}-2 \widetilde{A}\left(\xi, \xi^{\prime}\right)$ is equal to $d_{M}\left(\xi, \xi^{\prime}\right)^{2}$, the squared distance defined in (22). The distance function takes a minimum if and only if $\xi=\xi^{\prime}$, so that

$$
\exp \left(\frac{i}{2\left(t-t_{0}\right) \hbar} d_{M}\left(\xi, \xi^{\prime}\right)^{2}\right)
$$

makes a definite contribution as part of an integral kernel. Thus, for small $t-t_{0}$, one may conclude that the function $\widetilde{F}_{t}^{m}\left(\xi, \xi^{\prime}\right)$ has an asymptotic expansion of the form

$$
\widetilde{F}_{t}^{m}\left(\xi, \xi^{\prime}\right) \sim \frac{1}{\widetilde{A}\left(\xi, \xi^{\prime}\right)^{1 / 2}} \frac{1}{\left(2 \pi i\left(t-t_{0}\right) \hbar\right)^{3 / 2}} \exp \left(\frac{i}{2\left(t-t_{0}\right) \hbar} d_{M}\left(\xi, \xi^{\prime}\right)^{2}\right) .
$$

\section{REMARKS ON ELECTRONS ON A PLANE}

A system of electrons on a plane is of fundamental interest from the viewpoint of the quantum Hall effect. In his lecture ${ }^{17}$ on the quantum Hall effect, Laughlin gives a trial wave function for planar three electrons on a plane, which is expressed as

$$
\psi_{n, m}\left(z_{1}, z_{2}\right)=\left(\left(z_{2}+i z_{1}\right)^{3 m}-\left(z_{2}-i z_{1}\right)^{3 m}\right)\left(z_{1}^{2}+z_{2}^{2}\right)^{n} e^{-(1 / 4)\left(\left|z_{1}\right|^{2}+\left|z_{2}\right|^{2}\right)},
$$

up to a constant factor, where $z_{1}$ and $z_{2}$ are variables given in (9). It is easy to see that this function is equivariant under the $\mathrm{U}(1)$ action. We can also verify that the function $\psi_{n, m}$ satisfies the Pauli principle. To show this, we have only to see how $z_{2}+i z_{1}$ and $z_{2}-i z_{2}$ transform under the $S_{3}$ action. Writing out the transformation (29) for every element $h \in S_{3}$ results in the following transformation except for the identity:

$$
\begin{gathered}
\left(z_{2}+i z_{1}\right) \rho(12)^{-1}=z_{2}-i z_{1}, \quad\left(z_{2}-i z_{1}\right) \rho(12)^{-1}=z_{2}+i z_{1}, \\
\left(z_{2}+i z_{1}\right) \rho(13)^{-1}=\left(z_{2}-i z_{1}\right) e^{-(2 \pi / 3) i}, \quad\left(z_{2}-i z_{1}\right) \rho(13)^{-1}=\left(z_{2}+i z_{1}\right) e^{(2 \pi / 3) i}, \\
\left(z_{2}+i z_{1}\right) \rho(23)^{-1}=\left(z_{2}-i z_{1}\right) e^{(2 \pi / 3) i}, \quad\left(z_{2}-i z_{1}\right) \rho(23)^{-1}=\left(z_{2}+i z_{1}\right) e^{-(2 \pi / 3) i}, \\
\left(z_{2}+i z_{1}\right) \rho(123)^{-1}=\left(z_{2}+i z_{1}\right) e^{(2 \pi / 3) i}, \quad\left(z_{2}-i z_{1}\right) \rho(123)^{-1}=\left(z_{2}-i z_{1}\right) e^{-(2 \pi / 3) i}, \\
\left(z_{2}+i z_{1}\right) \rho(132)^{-1}=\left(z_{2}+i z_{1}\right) e^{-(2 \pi / 3) i}, \quad\left(z_{2}-i z_{1}\right) \rho(132)^{-1}=\left(z_{2}-i z_{1}\right) e^{(2 \pi / 3) i}
\end{gathered}
$$


From these transformations, it follows that $\psi_{m, n}$ indeed satisfies the Pauli principle under the $S_{3}$ action.

According to our procedure (60), we can form another function which satisfies the Pauli principle. For example, taking an equivariant function $\left(z_{2}+i z_{1}\right)^{m}$ as a seed, we can form a function

$$
\begin{gathered}
\left(z_{2}+i z_{1}\right)^{m}+\left(z_{2}+i z_{1}\right)^{m} e^{(2 m \pi / 3) i}+\left(z_{2}+i z_{1}\right)^{m} e^{-(2 m \pi / 3) i}-\left(z_{2}-i z_{1}\right)^{m} \\
-\left(z_{2}-i z_{1}\right)^{m} e^{(2 m \pi / 3) i}-\left(z_{2}-i z_{1}\right)^{m} e^{-(2 m \pi / 3) i}
\end{gathered}
$$

which is subject to the Pauli principle. We can multiply the function (113) by the factor $e^{-(1 / 4)\left(\left|z_{1}\right|^{2}+\left|z_{2}\right|^{2}\right)}$ to form a plausible wave function.

\section{ACKNOWLEDGMENT}

This work is supported partly by the Grant-In-Aid for Scientific Research of the Ministry of Education, Culture, Sports, Science and Technology of Japan (13640209).

${ }^{1}$ T. Iwai, J. Math. Phys. 28, 964 (1987).

${ }^{2}$ A. Guichardet, Ann. I.H.P. Phys. Theor. 40, 329 (1984).

${ }^{3}$ T. Iwai, J. Math. Phys. 28, 1315 (1987).

${ }^{4}$ T. Iwai, Ann. I.H.P. Phys. Theor. 47, 199 (1987).

${ }^{5}$ T. Iwai, J. Math. Phys. 29, 1325 (1988).

${ }^{6}$ T. Iwai, J. Phys. A 31, 3849 (1998).

${ }^{7}$ T. Iwai, J. Math. Phys. 40, 2381 (1999).

${ }^{8}$ T. Iwai and A. Tachibana, Ann. I.H.P. Phys. Theor. 70, 525 (1999).

${ }^{9}$ S. Tanimura and T. Iwai, J. Math. Phys. 41, 1814 (2000).

${ }^{10}$ A. Tachibana and T. Iwai, Phys. Rev. A 33, 2262 (1986).

${ }^{11}$ R. G. Littlejohn and M. Reinsch, Rev. Mod. Phys. 69, 213 (1997).

${ }^{12}$ F. Wilczek, in XVIIth International Colloquium on Group Theoretical Method in Physics, edited by Y. Saint-Aubin and L. Vinet (World Scientific, Singapore, 1989), pp. 154-167.

${ }^{13}$ R. Montgomery, in The Geometry of Hamiltonian Systems, edited by T. Ratiu (Springer, Berlin, 1991), pp. 403-438.

${ }^{14}$ R. Montgomery, in Dynamics and Control of Mechanical Systems, edited by M. J. Enos (American Mathematical Society, 1993), pp. 193-218.

${ }^{15}$ W. Ludwig and C. Falter, Symmetries in Physics (Springer, Berlin, 1988).

${ }^{16}$ K. A. Mitchell and R. G. Littlejohn, Phys. Rev. A 61, 042502 (2000).

${ }^{17}$ R. B. Laughlin, in The Quantum Hall Effect, edited by R. E. Prange and S. M. Girvin (Springer, New York, 1987). 